\title{
Erratum zu: Grenzen der symmetrischen Varianz
}

\section{Erratum zu:}

Kapitel 6 in: M. Hellwig, Partikelemissionskonzept und probabilistische Betrachtung der Entwicklung von Infektionen in Systemen, https://doi.org/10.1007/978-3-658-33157-3_6

Ein bedauerlicher Fehler im Verlag hat dazu geführt, dass die Inhalte in Kapitel 6 neu gesetzt wurden. Der Verlag hat dies nachträglich korrigiert.

Die korrigierte Version des Kapitels ist verfügbar unter https://doi.org/10.1007/978-3-658-33157-3_6 